\title{
Optimization of a Solid-Phase Extraction Protocol for Fractionation of Selected Steroids Using Retention Data from Micro Thin-layer Chromatography
}

\author{
Paweł K. ZARZYCKI, ${ }^{* \dagger}$ Elżbieta WŁodARCZYK, * Magdalena B. ZARZYCKA, ${ }^{*}$ and Bronisław K. GŁóD** \\ * Section of Toxicology and Bioanalytics, Department of Environmental Biology, \\ Koszalin University of Technology, Śniadeckich 2, 75-453 Koszalin, Poland \\ **Department of Analytical Chemistry, Institute of Chemistry, Faculty of Science, University of Podlasie, \\ 3 Maja 54, 08-110 Siedlce, Poland
}

\begin{abstract}
In this paper a simple protocol is described for estimating of solid-phase extraction (SPE) elution volumes of steroids based on retention data generated from micro-planar chromatography. Particularly, the retention of selected steroids, including estrogens and progestagens, was studied on wettable with water octadecylsilica HPTLC plates and mobile phases composed of methanol:water mixtures ranging from 20 to $100 \%(\mathrm{v} / \mathrm{v})$. It was found that TLC retention data can be linearized by plotting $R_{\mathrm{M}}$ values of steroids against a reciprocal form of the organic modifier molar fraction $\left(1 / X_{\mathrm{s}}\right)$. Using such a mathematical approach, the retention parameter of steroids investigated could be easily back-calculated for a wide range of mobile-phase compositions, using few initial experimental data points. The hold-up time of SPE cartridges filled with $0.5 \mathrm{~g}$ of $\mathrm{C}-18$ adsorbent was determined experimentally, and appropriate retention factor values $\left(k_{\mathrm{SPE}}\right)$ for components of interest studied were calculated. Using an appropriate slope and intercept coefficients of the linear-regression equation formed as $\log k_{\mathrm{SPE}}=a R_{\mathrm{M}}+b$, the steroids SPE elution volumes were predicted beyond the experimental data range that was available for a solid-phase extraction experiment, particularly for mobile phases that contained a high level of water.
\end{abstract}

(Received January 6, 2009; Accepted April 1, 2009; Published July 10, 2009)

Solid-phase extraction (SPE) is an effective analytical tool commonly used for the fractionation, preconcentration and purification of a component of interest from complex biological

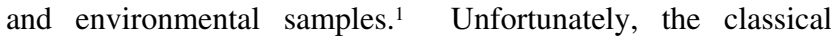
procedure of the SPE protocol optimization for the isolation of a number of analytes from multicomponent samples, particularly using the breakthrough curves method, can be expensive. This is because a quantitative amount of pure standards must be used. Moreover, such a procedure is usually time consuming, due to a number of fractions across a wide range of binary mobile phases for each analytical standard that should be collected and quantified. Therefore, an alternative method for the rapid computation of appropriate binary mixture concentrations and volumes for the purification and elution steps of a solid-phase extraction protocol based on e.g. the solvation parameter model have been proposed and tested., 2,3 On the other hand, the SPE cartridge can be considered as a short chromatographic column, and the retention of analytes in a such system should be simply predicted using retention data from HPLC experiment, in which a similar type of stationary phase and a wide range of solvent composition were studied. Recently, it has been suggested that planar chromatography may be used as a convenient pilot technique for estimating the SPE and HPLC behaviors of pesticide groups, separated under RP conditions. ${ }^{4,5}$ The main advantage of such an approach is that on the plate a number of samples can be analyzed simultaneously, and therefore appropriate retention data can be collected within a relatively short period of time compared to the SPE or HPLC techniques.

One of the advantages of modern HPTLC plates over classical

† To whom correspondence should be addressed.

E-mail: pkzarz@wp.pl; pawel_k_z@hotmail.com
TLC systems is that the eluent developing distance can be reduced to less than $50 \mathrm{~mm}$, even for separations involving a non-forced developing mode. This idea is based on an observation that the minimum values of the plate height $(H)$ can be achieved if the solvent migration distance along the HPTLC plate ranges from 30 to $40 \mathrm{~mm}^{6}$ Therefore, it can be expected that within and close to this migration distance range more dense spots can be obtained. In practice, under such conditions efficient separation for many types of compounds was observed, whereas the total analysis time was dramatically reduced compared to chromatographic separation performed on 10 or $20 \mathrm{~cm}$ long TLC plates. ${ }^{7-12}$

Previously, it had been demonstrated that micro thin-layer chromatography performed on the wettable with water RP18 plates and organic/water mobile phases, can be a useful technique for estimating the SPE behavior of estetrol, which was eluted from octadecylsilica packed cartridges. ${ }^{11}$ The main goals of present paper are to show the high separation throughput and capability of micro-planar chromatography as a fast and robust retention data source, as well as to demonstrate that this technique can be useful for an accurate prediction of the SPE behavior over a wide range of key steroids eluted from $\mathrm{C} 18$ tubes. The described protocol may be applied for a fast optimization of the solid-phase extraction procedure for the isolation, pre-purification and concentration of a complex steroids mixture including estrogens and progestagens.

\section{Experimental}

Reagents and chemicals

Steroid standards including estetrol and 20 $\alpha$-hydroxy- 


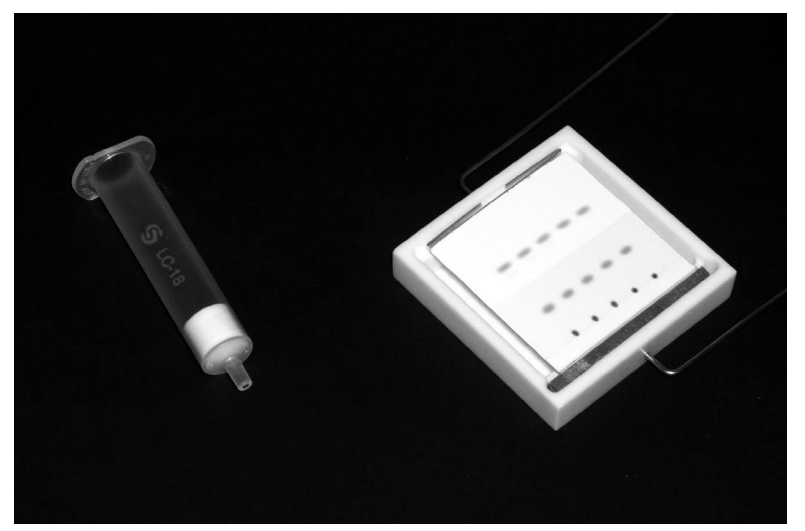

Fig. 1 SPE cartridge (left) and PTFE chamber unit with micro-TLC plate (right).

progesterone were purchased from Steraloids, London, England. Progesterone was obtained from Merck, Darmstadt, Germany. Equilin and $17 \alpha$-hydroxyprogesterone were products of Sigma St. Louis, MO. Estriol, $17 \alpha$-estradiol, $17 \beta$-estradiol and estrone were obtained from Aldrich Chemical Co., Milwaukee, WI. Methanol (LiChrosolv $99.8 \%$ for liquid chromatography) was a product of Merck. Double-distilled tap water was used for mobile-phase preparation. Phosphomolybdic acid was purchased from Chempur, Piekary Śląskie, Poland.

\section{Micro-planar chromatography}

A separation experiment was performed on glass-based HPTLC RP18W plates that were a product of Merck (Darmstadt, Germany). Before sample application, the factory-prepared plates $(100 \times 100 \mathrm{~mm})$ were cut to a working size of $50 \times$ $50 \mathrm{~mm}$. In each case, a sample starting line was placed $5 \mathrm{~mm}$ from the plate bottom edge, allowing a maximum eluent front migration distance of $45 \mathrm{~mm}$. Micro-planar separations were performed using a home-made temperature-controlled removable horizontal micro-TLC chamber unit, described previously. ${ }^{11}$ Particularly, a chromium-coated brass unit was working inside a foam insulated metal oven connected to an external liquid circulating thermostat Neslab RTE7 (Thermo Electron Co., Newington, USA). The system provided a constant TLC plate temperature, which was set at $20^{\circ} \mathrm{C}$ with an accuracy of $\pm 0.05^{\circ} \mathrm{C}$.

To obtain chromatograms, the following chamber working protocol was applied. A TLC plate with steroids spotted on the starting line $(1 \mu \mathrm{L}$ of each steroid stock solution at concentration of $1 \mathrm{mg} \mathrm{mL}^{-1}$ was applied) was positioned horizontally inside a chamber module with the stationary phase layer placed up side down. Afterward, the chamber module was transferred into a thermostating oven cavity and sealed using a 1-mm thin glass cover. Then, the movable cover of the oven was slid so as to reach the position above the TLC chamber module, and the temperature as well as the mobile phase equilibration step was performed for $15 \mathrm{~min}$. To obtain saturated chamber conditions, the unit saturation tray was filled with $5 \mathrm{~mL}$ of the mobile phase. The main chromatographic process was started after injecting a given eluent in a volume of $1 \mathrm{~mL}$ through an injection pipe into a mobile-phase application bar. Finally, the TLC plate was removed from the chamber module immediately after the mobile-phase front reached the plate edge located opposite to the application bar.

Chromatographic separation was performed under saturated chamber conditions for a wide range of methanol:water
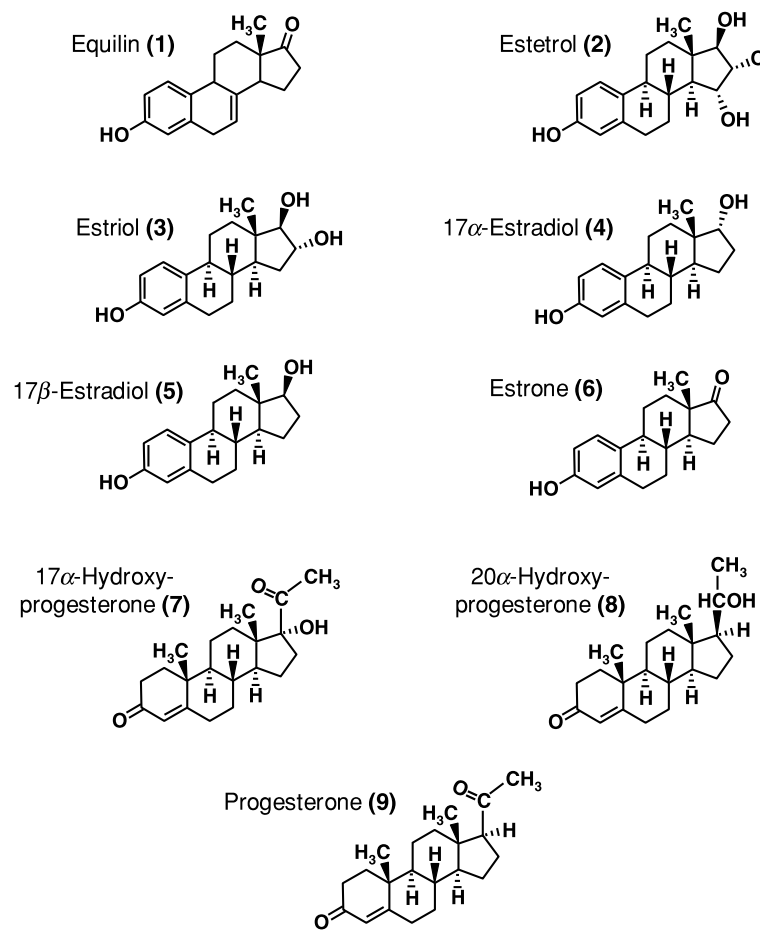

Fig. 2 Chemical structures of investigated steroids.

concentrations $(0-100 \%, v / v)$. Steroids spots were located using a visualization reagent consisted of $10 \%$ phosphomolybdic acid (PMA) in methanol. Blue/grey spots of steroids were generated after the plates were dipped in the PMA reagent and heating at $100^{\circ} \mathrm{C}$ for $10 \mathrm{~min}$.

\section{Solid-phase extraction protocol}

A solid-phase extraction (SPE) experiment was conducted using octadecylsilica packed tubes LC-18 SPE (Supelclean, $6 \mathrm{~mL}, 0.5 \mathrm{~g}$ ) obtained from Supelco, Bellefonte, PA. Steroid breakthrough curves were determined using methanol:water mobile phases. The protocol involved SPE tube conditioning, which was $5 \times 1 \mathrm{~mL}$ of $100 \%$ methanol, and then $5 \times 1 \mathrm{~mL}$ of the elution solvent ranging from 30 to $100 \%(\mathrm{v} / \mathrm{v})$ methanol. The stationary phase in the tube was allowed to dry for $1 \mathrm{~min}$ using a vacuum pump, and then $10 \mu \mathrm{g}$ of a steroid standard ( $20 \mu \mathrm{L}$ of $500 \mu \mathrm{g} \mathrm{mL}^{-1}$ in methanol) was added. The tube was dried for $2 \mathrm{~min}$, and each sample was eluted with $1 \mathrm{~mL}$ steps using methanol concentrations ranging from 30 to $100 \%$. Each $1 \mathrm{~mL}$ volume of the eluted sample was quantified by UV spectrophotometry..$^{13}$

The hold-up volume $\left(V_{\mathrm{o}}\right)$ of the SPE cartridges was determined by weighting an SPE tube sorbent that was dried and wetted with methanol and water. Due to a strong peak asymmetry of the steroids eluted under the solid-phase extraction conditions, the SPE retention parameter $\left(k_{\mathrm{SPE}}=\left(V-V_{\mathrm{o}}\right) / V_{\mathrm{o}}\right)$ was based on the whole elution volume of the individual steroid, instead of the elution volume estimated using the peak maximum criterion.

\section{Chromatograms digitalization and image data proceeding}

Developed and visualized with PMA micro-chromatograms were scanned under visible light conditions with an 8-bit per RGB channel color deep mode, and saved as TIFF files without compression. Picture acquisition was performed using a Plustek OpticPro S12 USB scanner (Plustek, Taipei, Taiwan) with the 
Table 1 Micro-TLC $\left(R_{\mathrm{F}}\right)$ and SPE $\left(k_{\mathrm{SPE}}\right)$ data for investigated steroids obtained from RP18W HPTLC plates and C-18 tubes using methanol:water $(\%, \mathrm{v} / \mathrm{v})$ mobile phases

\begin{tabular}{|c|c|c|c|c|c|c|c|c|c|c|c|c|c|c|c|}
\hline \multirow{2}{*}{ Steroid } & \multirow{2}{*}{$\begin{array}{l}\text { Retention } \\
\text { parameter }\end{array}$} & \multicolumn{14}{|c|}{ Methanol/water, \% (v/v) } \\
\hline & & 20 & 30 & 35 & 40 & 50 & 55 & 58 & 60 & 65 & 70 & 80 & 85 & 90 & 100 \\
\hline \multirow[t]{2}{*}{ Equilin } & $R_{\mathrm{F}}$ & & & 0.02 & 0.03 & 0.10 & 0.14 & 0.19 & 0.21 & 0.28 & 0.35 & 0.48 & 0.50 & 0.54 & 0.58 \\
\hline & $k_{\mathrm{SPE}}$ & & & & & & 31.9 & & 20.9 & & 10.0 & 6.3 & & 4.5 & 2.7 \\
\hline \multirow[t]{2}{*}{ Estetrol } & $R_{\mathrm{F}}$ & 0.03 & 0.07 & 0.11 & 0.17 & 0.30 & 0.37 & 0.41 & 0.44 & 0.50 & 0.54 & 0.58 & 0.57 & 0.58 & 0.60 \\
\hline & $k_{\mathrm{SPE}}$ & & 46.5 & 26.4 & 15.4 & 8.1 & & & 4.5 & & 2.7 & 0.8 & & 0.8 & 0.8 \\
\hline \multirow[t]{2}{*}{ Estriol } & $R_{\mathrm{F}}$ & & 0.03 & 0.05 & 0.09 & 0.19 & 0.27 & 0.31 & 0.34 & 0.41 & 0.46 & 0.54 & 0.55 & 0.57 & 0.59 \\
\hline & $k_{\mathrm{SPE}}$ & & & & 42.9 & 17.3 & & & 6.3 & & 4.5 & 2.7 & & 0.8 & 0.8 \\
\hline \multirow[t]{2}{*}{$17 \alpha$-Estradiol } & $R_{\mathrm{F}}$ & & & 0.01 & 0.03 & 0.07 & 0.12 & 0.15 & 0.18 & 0.25 & 0.33 & 0.45 & 0.49 & 0.53 & 0.57 \\
\hline & $k_{\mathrm{SPE}}$ & & & & & & & 42.9 & 24.6 & & 10 & 6.3 & & 4.5 & 2.7 \\
\hline \multirow[t]{2}{*}{$17 \beta$-Estradiol } & $R_{\mathrm{F}}$ & & & 0.01 & 0.02 & 0.06 & 0.10 & 0.14 & 0.16 & 0.23 & 0.29 & 0.43 & 0.47 & 0.51 & 0.56 \\
\hline & $k_{\mathrm{SPE}}$ & & & & & 90.4 & 41 & & 15.5 & & 8.1 & 4.5 & & 2.7 & 0.8 \\
\hline \multirow[t]{2}{*}{ Estrone } & $R_{\mathrm{F}}$ & & & 0.01 & 0.03 & 0.08 & 0.13 & 0.17 & 0.20 & 0.27 & 0.34 & 0.47 & 0.51 & 0.53 & 0.57 \\
\hline & $k_{\mathrm{SPE}}$ & & & & & 50.2 & & & 20.9 & & 11.8 & 6.3 & & 4.5 & 2.7 \\
\hline \multirow{2}{*}{$\begin{array}{l}17 \alpha \text {-Hydroxy- } \\
\text { progesterone }\end{array}$} & $R_{\mathrm{F}}$ & & & 0.01 & 0.02 & 0.08 & 0.12 & 0.16 & 0.19 & 0.26 & 0.33 & 0.45 & 0.50 & 0.52 & 0.56 \\
\hline & $k_{\mathrm{SPE}}$ & & & & & & & & 31.9 & & 17.3 & 10.0 & 8.1 & 6.3 & 4.5 \\
\hline \multirow{2}{*}{$\begin{array}{l}20 \alpha \text {-Hydroxy- } \\
\text { progesterone }\end{array}$} & $R_{\mathrm{F}}$ & & & & 0.01 & 0.03 & 0.04 & 0.07 & 0.10 & 0.14 & 0.20 & 0.35 & 0.41 & 0.46 & 0.53 \\
\hline & $k_{\mathrm{SPE}}$ & & & & & & & & & & 31.9 & 13.6 & & 6.3 & 2.7 \\
\hline \multirow[t]{2}{*}{ Progesterone } & $R_{\mathrm{F}}$ & & & & 0.01 & 0.04 & 0.06 & 0.08 & 0.11 & 0.16 & 0.23 & 0.36 & 0.42 & 0.47 & 0.53 \\
\hline & $k_{\mathrm{SPE}}$ & & & & & & & & & 46.5 & 31.9 & 11.8 & & 6.3 & 4.5 \\
\hline
\end{tabular}

Capacity factor values were calculated using the SPE tube hold-up volume estimated at a level of $V_{0}=0.547 \mathrm{~mL}$ and mobile phase volumes according to a reference..$^{13}$

help of image-acquisition software: Image Folio Ver. 4.2.0; (1991 - 2000 NewSoft Technology Co.). An appropriate TLC plate area was cropped from the original frame size, and then a gray-scale conversion filter was applied. From selected crosssections of the chromatographic lanes that were extracted from the digital pictures with the help of Scion Image freeware (Scion Co.; Ver. 4.0.3.2; http://www.scioncorp.com/) retention factor $\left(R_{\mathrm{F}}\right)$ values of steroid spots were derived.

\section{Results and Discussion}

The main goal of our study was to test in practice an idea that solid-phase extraction elution volumes of low molecular mass compounds can be simply predicted using retention data from micro-planar chromatography. Particularly, the experiment involves SPE cartridges filled with $0.5 \mathrm{~g}$ of the C-18 adsorbent as well as $5 \times 5 \mathrm{~cm}$ HPTLC water-tolerable octadecylsilica plates developed using a micro-TLC chamber unit (Fig. 1). As components of interest, a number of steroids, including estrogens (equilin, estetrol, estriol, $17 \alpha$-estradiol, 17 $\beta$-estradiol and estrone) as well as progestagens (progesterone and its $17 \alpha$ - and $20 \alpha$-hydroxy derivatives) were investigated (Fig. 2). The selection of components of interest was based on their importance for metabolic studies and medical diagnostics. ${ }^{14}$ Table 1 contains retention data generated from micro-planar chromatography and expressed as the $R_{\mathrm{F}}$ values. $R_{\mathrm{F}}$ (retardation factor) is defined as the $a / b$ ratio, where $a$ denotes the spot migration distance from the plate start line and $b$ corresponds to the migration distance of the eluent front. As can be seen from graph A, which is presented in Fig. 3, under reversed-phase conditions, typical S-shaped curves are formed to investigated steroids, if the $R_{\mathrm{F}}$ values were plotted against $\%(\mathrm{v} / \mathrm{v})$ of methanol in water as the eluents. ${ }^{5,15}$ In order to find a linear fit for the obtained retention data, the $R_{\mathrm{M}}$ values were plotted against the eluent composition, expressed as \% (v/v), $X_{\mathrm{s}}$ and $\log X_{\mathrm{s}}{ }^{15,16}$ The chromatographic retention parameter $R_{\mathrm{M}}$ was introduced by Soczewiński and Wachtmeistes in 1962, and is
A
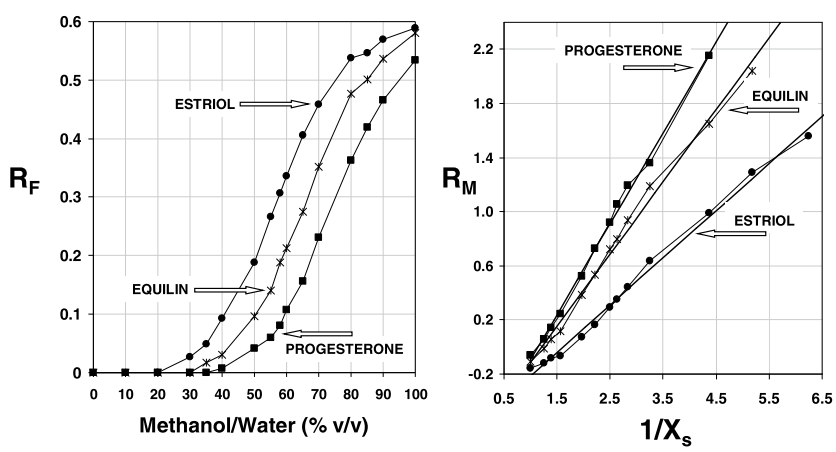

Fig. $3 R_{\mathrm{F}}$ and $R_{\mathrm{M}}$ values of selected steroids plotted against the mobile-phase composition, expressed as \% of methanol in water (A) and a reciprocal form of the organic modifier molar fraction (B).

extensively used for quantitative retention-solvent composition relationships as well as quantitative structure-activity relationships studies involving TLC retention data. ${ }^{15,17} R_{\mathrm{M}}$ values can be simply calculated from $R_{\mathrm{F}}$ values using the relationship $R_{\mathrm{M}}=\log \left[\left(1 / R_{\mathrm{F}}\right)-1\right]$, or directly derived from a planar chromatogram as a logarithmic form of the cla ratio, where $c$ denotes the distance between the spot and eluent front, while the distance $a$ is defined as described above.

Considering the whole range of experimental points available, it has been found that our data could be linearized by plotting relevant $R_{\mathrm{M}}$ values against the reciprocal form of the organic modifier molar fraction $\left(1 / X_{\mathrm{s}}\right)$, as shown in graph B (Fig. 3), while a linear fit using the above listed eluent data transformations was largely unsuccessful. Therefore, by using a regression coefficient corresponding to the equation formed as $R_{\mathrm{M}}=$ slope $\left(1 / X_{\mathrm{s}}\right)+$ intercept (Table 2$)$, the steroids retention within a wide range of mobile phase concentrations could be accurately estimated, and used for further investigations. It should be noted that based on such a mathematical approach the retention 
Table 2 Regression coefficients and determination coefficient $\left(r^{2}\right)$ of the equation formed as $R_{\mathrm{M}}=\operatorname{slope}\left(1 / X_{\mathrm{s}}\right)+$ intercept; calculated for steroids chromatographed under reversed-phase micro-HPTLC conditions using methanol-water mobile phases

\begin{tabular}{lccccccc}
\hline \multicolumn{1}{c}{ Steroid } & Concentration range, $\%(\mathrm{v} / \mathrm{v})$ & $n$ & Slope & Standard error & Intercept & Standard error & $r^{2}$ \\
\hline Equilin & $35-100$ & 12 & 0.48 & 0.01 & -0.65 & 0.03 & 0.994 \\
Estetrol & $20-100$ & 14 & 0.22 & 0.01 & -0.39 & 0.06 & 0.955 \\
Estriol & $30-100$ & 13 & 0.349 & 0.008 & -0.56 & 0.03 & 0.994 \\
$17 \alpha$-Estradiol & $35-100$ & 12 & 0.57 & 0.02 & -0.77 & 0.04 & 0.993 \\
$17 \beta$-Estradiol & $35-100$ & 12 & 0.53 & 0.01 & -0.64 & 0.04 & 0.994 \\
Estrone & $35-100$ & 12 & 0.50 & 0.01 & -0.66 & 0.03 & 0.994 \\
$17 \alpha$-Hydroxy-progesterone & $35-100$ & 12 & 0.58 & 0.01 & -0.79 & 0.04 & 0.995 \\
$20 \alpha$-Hydroxy-progesterone & $40-100$ & 11 & 0.68 & 0.02 & -0.74 & 0.05 & 0.992 \\
Progesterone & $40-100$ & 11 & 0.67 & 0.01 & -0.77 & 0.03 & 0.997 \\
\hline
\end{tabular}

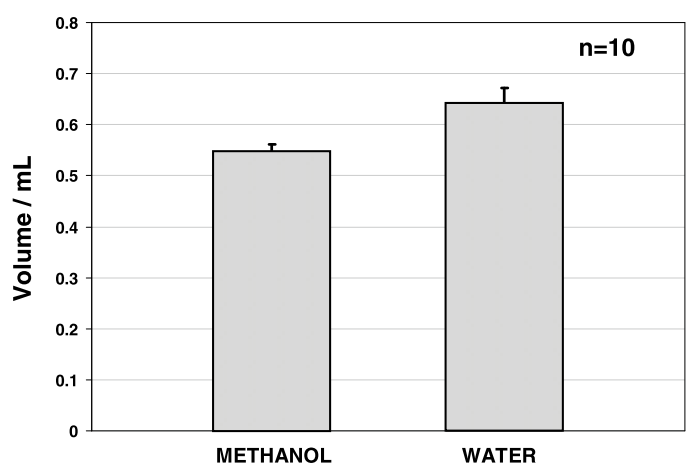

Fig. 4 Comparison of the SPE tubes dead volume $\left(V_{\mathrm{o}}\right)$ measured for methanol and water eluents; cartridge type, Supelclean LC-18; adsorbent mass, $0.5 \mathrm{~g}$.

parameter of the investigated steroids could be easily back-calculated for a wide range of mobile-phase compositions, using few initial experimental data points.

In principles, a typical SPE cartridge may work as a short chromatographic column, and therefore the hold-up volume $\left(V_{\mathrm{o}}\right)$ of such a separation system can be evaluated. Particularly, this parameter was determined by weighting the SPE tube sorbent, which was dried and wetted with methanol or water (Fig. 4). Based on such data, the values of the chromatographic retention parameter, $k_{\mathrm{SPE}}=\left(V-V_{\mathrm{o}}\right) / V_{\mathrm{o}}$ of analytes studied using the SPE system, could be also calculated (Table 1). Contrary to the micro-TLC experiment, the determination of the steroid retention parameters from an SPE elution curve is usually time consuming, due to a number of fractions that should be analyzed to get the elution profiles of the components of interest for each point of the eluent composition (Fig. 5).

According to data reported in the literature, the column chromatographic behavior of low molecular mass analytes, such as pesticides or coumarins can be estimated using retention data from the TLC experiment. ${ }^{4,5,15,16}$ This can be performed via the $R_{\mathrm{M}}=\log k$ relationship while taking into account that a similar mobile/stationary phase system is applied. The result of our study revealed that a linear relationship between the $R_{\mathrm{M}}$ parameter and the logarithmic form of the SPE retention factor $\left(\log k_{\mathrm{SPE}}\right)$ was observed for all of components of interest investigated (Table 3). However, the values of the SPE volumes, which are directly backcalculated from the $R_{\mathrm{M}}=\log k$ equation, may be affected by a strong underestimation of the eluent volume, particularly for a mobile phases containing a high level of water. As can be seen from the graphs presented in Fig. 6, the SPE elution volumes could be accurately predicted using an

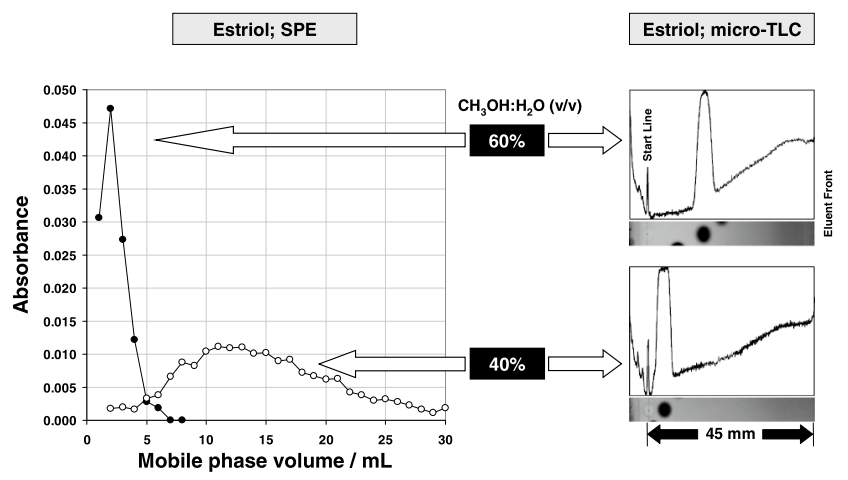

Fig. 5 Typical elution profiles of estriol from RP18 SPE cartridge and corresponding micro-TLC chromatograms of this steroid on RP18W plates. In both methods experimental data were obtained using eluents composed of 40 and $60 \%$ methanol in water (v/v).

appropriate slope and intercept coefficients of the linear regression equation $\log k_{\mathrm{SPE}}=a R_{\mathrm{M}}+b$ listed in Table 3B. It is noteworthy that under such conditions the SPE elution volumes, which are backcalculated from an appropriate linear-regression equation, can be properly estimated beyond the experimental data range available from the SPE experiment.

Micro-planar chromatography shows a great capability as a fast and robust retention data source. For the steroid group investigated and under reversed-phase conditions, a linear behavior between the TLC retention parameter, expressed as $R_{\mathrm{M}}$ values and mobile-phase composition transformed into reciprocal form of the organic modifier molar fraction $\left(1 / X_{\mathrm{s}}\right)$, was observed for a whole range of studied mobile-phase concentrations.

A linear relationship was observed between the $R_{\mathrm{M}}$ values of steroids plotted against the logarithmic form of the SPE retention factor $\left(k_{\mathrm{SPE}}\right)$. The SPE elution volumes of investigated steroids can be simply predicted using retention data from micro-planar chromatography via an equation formed as $\log k_{\mathrm{SPE}}=a R_{\mathrm{M}}+b$.

As the main advantages of the proposed approach, the protocol simplicity and fast data generation should be listed. It should also be noted that by using micro-TLC data the SPE elution volumes can be calculated beyond the SPE experimental data range available, particularly for mobile phases composed of a high level of water.

\section{Acknowledgements}

The experimental work was supported by the Ministry of Education and Science, Poland (Grant No. N 523043 32/1477). 
Table 3 Relationship between SPE and micro-TLC data (slope $a$ and intercept $b$ coefficients of the linear-regression equation (log $k_{\mathrm{SPE}}=$ $a R_{\mathrm{M}}+b$ ) were calculated from $\log k_{\mathrm{SPE}}$ and $R_{\mathrm{M}}$ data using whole (A) and selected (B) mobile-phase concentrations)

\begin{tabular}{|c|c|c|c|c|c|c|c|}
\hline Steroid & Concentration range, $\%(\mathrm{v} / \mathrm{v})$ & $n$ & Slope & Standard error & Intercept & Standard error & $r^{2}$ \\
\hline \multicolumn{8}{|l|}{ (A) } \\
\hline Equilin & $55-100$ & 6 & 1.09 & 0.09 & 0.68 & 0.04 & 0.976 \\
\hline Estetrol & $30-100$ & 9 & 1.3 & 0.1 & 0.28 & 0.07 & 0.936 \\
\hline Estriol & $40-100$ & 7 & 1.4 & 0.2 & 0.32 & 0.09 & 0.923 \\
\hline $17 \alpha$-Estradiol & $58-100$ & 6 & 1.24 & 0.09 & 0.65 & 0.04 & 0.979 \\
\hline $17 \beta$-Estradiol & $50-100$ & 7 & 1.4 & 0.1 & 0.32 & 0.09 & 0.957 \\
\hline Estrone & $50-100$ & 6 & 1.03 & 0.09 & 0.69 & 0.05 & 0.968 \\
\hline $17 \alpha$-Hydroxy-progesterone & $60-100$ & 6 & 1.1 & 0.1 & 0.86 & 0.03 & 0.967 \\
\hline $20 \alpha$-Hydroxy-progesterone & $70-100$ & 4 & 1.6 & 0.2 & 0.61 & 0.08 & 0.956 \\
\hline Progesterone & $65-100$ & 5 & 1.34 & 0.08 & 0.74 & 0.03 & 0.991 \\
\hline \multicolumn{8}{|l|}{ (B) } \\
\hline Equilin & $55-90$ & 5 & 1.00 & 0.03 & 0.74 & 0.02 & 0.997 \\
\hline Estetrol & $30-70$ & 6 & 0.99 & 0.03 & 0.53 & 0.02 & 0.997 \\
\hline Estriol & $40-80$ & 5 & 1.12 & 0.05 & 0.52 & 0.03 & 0.994 \\
\hline $17 \alpha$-Estradiol & $60-90$ & 4 & 1.04 & 0.05 & 0.7 & 0.02 & 0.996 \\
\hline $17 \beta$-Estradiol & $50-90$ & 6 & 1.22 & 0.08 & 0.44 & 0.06 & 0.984 \\
\hline Estrone & $50-90$ & 5 & 0.94 & 0.05 & 0.75 & 0.03 & 0.991 \\
\hline $17 \alpha$-Hydroxy-progesterone & $60-90$ & 5 & 1.02 & 0.08 & 0.89 & 0.02 & 0.984 \\
\hline $20 \alpha$-Hydroxy-progesterone & $70-90$ & 3 & 1.3 & 0.1 & 0.73 & 0.06 & 0.988 \\
\hline Progesterone & $65-90$ & 4 & 1.3 & 0.1 & 0.74 & 0.05 & 0.985 \\
\hline
\end{tabular}

A
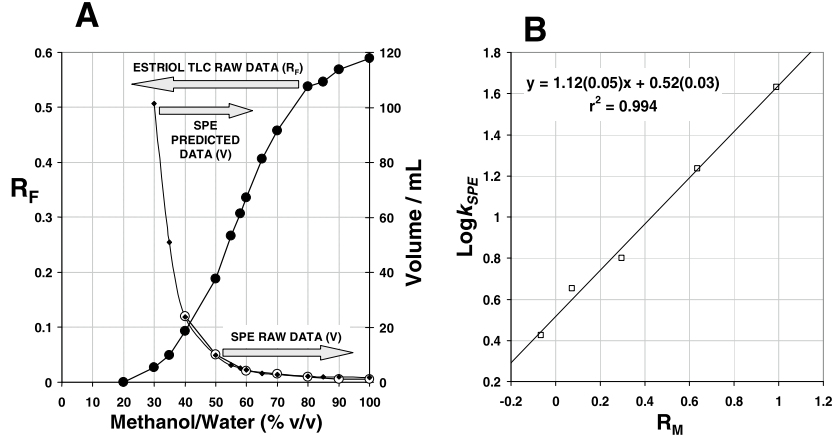

Fig. 6 Estimation of the estriol elution volumes from an SPE tube, using micro-TLC data. Circles and black dots on graph A correspond to the real elution data points obtained from SPE and micro-TLC experiments, respectively. Small black diamonds represent a back-calculated SPE elution volumes curve of estriol, using the micro-TLC data via $\log k_{\mathrm{SPE}}=a R_{\mathrm{M}}+b$ equation (graph $\left.\mathrm{B}\right)$.

\section{References}

1. B. Tippins, Nature, 1988, 334, 273.

2. R. J. Ozretich and W. P. Schroeder, Anal. Chem., 1986, 58, 2041.

3. D. S. Seibert and C. F. Poole, J. High. Resolut. Chromatogr., 1998, $21,481$.

4. T. Tuzimski and E. Soczewiński, Chromatographia, 2002, $56,225$.
5. T. Tuzimski and E. Soczewiński, in "Problems of Science, Teaching and Therapy", 2002, No. 12, Medical University of Lublin, Lublin, Poland.

6. P. E. Wall, "Thin-layer Chromatography. A Modern Practical Approach", 2006, Springer-Verlag, RSC, Cambridge.

7. E. Reich, A. Blatter, and B. Meier, "TLC for the Analysis of Herbal Drugs", 2003, Camag Scientific Note, Muttenz, available at: http://www.camag.ch/index.php.

8. C. F. Poole and S. K. Poole, "Chromatography Today", 1991, Elsevier, Amsterdam.

9. L. S. Litvinova and V. N. Zgonnik, J. Planar Chromatogr., 1997, 10, 38.

10. P. K. Zarzycki, H. Ohta, F. B. Harasimiuk, and K. Jinno, Anal. Sci., 2007, 23, 1391.

11. P. K. Zarzycki, J. Chromatogr., A, 2008, 1187, 250.

12. P. K. Zarzycki and M. B. Zarzycka, Anal. Bioanal. Chem., 2008, 391, 2219.

13. P. K. Zarzycki, K. M. Kulhanek, R. Smith, and V. L. Clifton, J. Chromatogr., A, 2006, 1104, 203.

14. V. L. Clifton, A. Bisits, and P. K. Zarzycki, J. Chromatogr., $B, \mathbf{2 0 0 7}, 855,249$.

15. E. Soczewiński, in "A Century of Separation Science: Quantitative Retention-Eluent Composition Relationships in Partition and Adsorption Chromatography", ed. H. J. Issaq, 2002, Marcel Dekker, New York.

16. M. A. Hawryl, E. Soczewiński, and T. H. Dzido, J. Chromatogr., A, 2000, 886, 75.

17. E. Soczewiński and C. A. Wachtmeister, J. Chromatogr., 1962, 7, 311. 